\title{
OPEN Maternal separation with early weaning impairs neuron-glia integrity: non-invasive evaluation and substructure demonstration
}

\begin{abstract}
Haiyan Zeng ${ }^{1,2}$, Xiaolei Zhang ${ }^{3}$, Wenqiang Wang ${ }^{2}$, Zhiwei Shen ${ }^{3}$, Zhuozhi Dai $^{3}$, Zhijia Yu $^{1}$, Shuqin $\mathrm{Xu}^{4}$, Gen Yan ${ }^{3}$, Qingjun Huang ${ }^{1}$, Renhua Wu ${ }^{3}$, Xi Chen ${ }^{5}$ \& Haiyun Xu ${ }^{1}{ }^{1,4,6 \bowtie}$

Astrocytes and oligodendrocytes play essential roles in regulating neural signal transduction along neural circuits in CNS. The perfect coordination of neuron/astrocyte and neuron/oligodendrocyte entities was termed as neuron-glia integrity recently. Here we monitored the status of neuron-glia integrity via non-invasive neuroimaging methods and demonstrated the substructures of it using other approaches in an animal model of maternal separation with early weaning (MSEW), which mimics early life neglect and abuse in humans. Compared to controls, MSEW rats showed higher glutamate level, but lower GABA in prefrontal cortex (PFC) detected by chemical exchange saturation transfer and ${ }^{1} H$-MRS methods, lower levels of glial glutamate transporter-1 and ATP- $\alpha$, but increased levels of glutamate decarboxylase- 65 and glutamine synthetase in PFC; reduced fractional anisotropy in various brain regions revealed by diffusion tensor imaging, along with increased levels of $\mathrm{N}$-acetylaspartate measured by ${ }^{1} \mathrm{H}-\mathrm{MRS}$; and hypomyelination in PFC as evidenced by relevant cellular and molecular changes.
\end{abstract}

Brain cells are classified into neurons and glial cells including astrocytes, microglia, and oligodendrocytes (OLs). Neurons have been thought of as principal cells in the brain because they receive and transmit chemical and electrical signals. Neurons connect each other thereby constituting neuronal chains known as neural circuits through which electrical signals propagate in the central nervous system (CNS). Glial cells have been regarded as supporting cells in the mammalian brain for many decades. However, increasing evidence suggests diverse and dynamic functions of glial cells ${ }^{1}$. For instance, astrocytes contact with synapses between neurons and regulate synaptic transmission as the third part of the tripartite synapse $\mathrm{e}^{2,3}$. OLs wrap neuronal axons and form the myelin sheath in CNS. The compacted myelin sheath provides high electrical resistance and low capacitance essential for saltatory impulse propagation ${ }^{4}$. Now it is believed that astrocyte and neuron function as an integrative entity thereby signal transmission within CNS is adjusted ${ }^{5,6}$. And axon-myelin co-works as an integrative entity that ensures partly processed information can be properly synchronized at post-synaptic sites. This synchronization is critical for information processing associated with perception, thought and action ${ }^{7,8}$. Based on these advances in neuron-glia communication research, we developed the neuron-glia integrity theory to highlight the precise regulation of neural signal transmission in CNS by glia cells 9 .

According to the neuron-glia integrity theory, the glutamate-glutamine-GABA cycle functions as the neurochemical substrates of neuron-astrocyte entity. Any changes in glutamate (Glu), glutamine (Gln), and/or GABA in a brain region are indicative of an imbalanced Glu-Gln-GABA cycle or impaired neuron-astrocyte entity there. On the other side, N-acetyl-aspartate (NAA) is considered the neurochemical correlate of axon-myelin entity because it involves in myelination and axon-glial signaling, in addition to a role in osmoregulation ${ }^{9,10}$. All these brain metabolites (Glu, Gln, GABA, and NAA), along with the others, can be measured and quantified in living subjects by means of non-invasive neuroimaging methods including proton magnetic resonance spectroscopy $\left({ }^{1} \mathrm{H}-\mathrm{MRS}\right)^{11}$ and chemical exchange saturation transfer $(\mathrm{CEST})^{12}$. As such, we proposed in a recent article to evaluate the functional status of neuron-glia entities in living subjects by means of non-invasive neuroimaging

\footnotetext{
${ }^{1}$ The Mental Health Center, Shantou University Medical College, Shantou, China. ${ }^{2}$ Xianyue Hospital/Xiamen Mental Health Center, Xiamen, China. ${ }^{3}$ Department of Medical Imaging, The Second Affiliated Hospital, Shantou University Medical College, Shantou, China. " $D$ epartment of Anatomy, Shantou University Medical College, Shantou, China. ${ }^{5}$ McLean Imaging Center, McLean Hospital, Harvard Medical School, Belmont, USA. ${ }^{6}$ The School of Psychiatry, Wenzhou Medical University, Wenzhou, China. ${ }^{\circledR}$ email: hyxu@stu.edu.cn
} 
techniques. In animal studies, the substructures of neuron-glia integrity in cellular and molecular levels can be demonstrated with brain samples ${ }^{9}$. Therefore, it is plausible to relate the neuron-glia entity status to higher brain function. The goals of this study were to evaluate the neuron-glia integrity in living rats by non-invasive neuroimaging methods and to demonstrate the substructure of neuron-astrocyte and neuron-OL entities at cellular and molecular levels.

To accomplish the above goals and to pave the way to the application of the integrity theory to future clinical research, we modified the MSEW paradigm designed by George and colleagues ${ }^{13}$ and applied it to Sprague-Dawley (S-D) rats. The MSEW paradigm was used to mimic early life neglect and abuse in humans as this paradigm is believed to influence brain development and consequently bring forth a predisposition toward mental and behavioral disorders ${ }^{14}$. For the first time we measured the integrity of neuron-glia entities in brains of living rats by non-invasive neuroimaging techniques of ${ }^{1} \mathrm{H}-\mathrm{MRS}$, CEST of glutamate (GluCEST), and diffusion tensor imaging (DTI). ${ }^{1} \mathrm{H}$-MRS has been extensively used in clinical practice and psychiatric research ${ }^{11}$, but no prior studies applied it to evaluate the neuron-glia entities. GluCEST is a recently developed technique to image glutamate. It was successfully used to map the Glu signal in healthy human brain by Cai et al. ${ }^{12}$ and has since been applied to animal models of neurological disorders, including Alzheimer's disease ${ }^{15,16}$, Huntington's disease ${ }^{17}$, epilepsy ${ }^{18}$, and multiple sclerosis ${ }^{19,20}$, but few applications were published in psychiatry research ${ }^{21}$. DTI is an MRI modality able to assess the micro-structural features of white matter and microfiber pathways by measuring the diffusional motion of water molecules, but no prior studies combined DTI and ${ }^{1} \mathrm{H}$-MRS methods to demonstrate the validity of NAA being used as the specific neurochemical correlate of axon-myelin entity. Moreover, by means of cellular and molecular methods as well as the non-invasive neuroimaging approaches, we demonstrated the cellular and molecular underpinnings of impaired neuron-astrocyte and neuron-OL entities in MSEW rats. In addition, this study presented the behavioral data from open field test that measures locomotor activity, exploratory behavior, and anxiety level of subjects.

\section{Results}

MSEW increased explorative activity and anxiety levels in rats. After the MSEW procedure, rats continued to live as those in Control group. On postnatal day 60 (PD 60), rats in the MSEW and Control groups were subjected to open field test to measure their locomotor activity, exploratory behavior, and anxiety level. It was found: (1) MSEW rats moved a longer distance in whole arena (Fig. 1A) of the open field compared to Control group $(6685.50 \pm 874.12 \mathrm{~cm}$ vs $5331.59 \pm 723.86 \mathrm{~cm}, p<0.001)$; (2) MSEW rats moved at a higher speed (Fig. 1B) compared to Control group $(11.24 \pm 1.41 \mathrm{~cm} / \mathrm{s}$ vs $9.04 \pm 1.17 \mathrm{~cm} / \mathrm{s}, p<0.001)$; (3) MSEW rats moved a shorter distance on the central zone (Fig. 1C) compared to Control group $(380.64 \pm 94.07 \mathrm{~cm}$ vs $462.46 \pm 64.07 \mathrm{~cm}, p<0.01$ ); and (4) MSEW rats spent less time on the central zone (Fig. 1D) compared to Control group $(21.36 \pm 6.69$. $s$ vs $27.31 \pm 3.93 \mathrm{~s}, p<0.01)$. These data indicate the presence of higher levels of exploratory activity and anxiety in the MSEW rats relative to Control group.

To examine possible malnutritional effects of MSEW procedure on rats, the body weight of rats was weighed on PDs 7, 14, 21, and 30, respectively. MSEW rats were same as those in Control group in body weight measured at all the time points (Supplementary Fig. 1), indicating the paradigm did not cause malnutrition in rat pups.

MSEW elevated global GluCEST contrast in the brain and increased Glu levels in prefrontal cortex and hippocampus of rats. GluCEST imaging has been used to map spatial distribution of Glu in the brain with a good spatial resolution in previous studies ${ }^{12,17,22}$. Referring to the protocol described in the previous studies, effects of MSEW on Glu distribution in rat brain were examined using GluCEST in this study. Before GluCEST imaging, CEST phantoms were prepared and scanned to optimize CEST and MRI procedures. The results showed that the GluCEST contrast was linearly proportional to Glu concentration in the physiological range at $\mathrm{pH}=7.0$ (Supplementary Fig. 2), but the other metabolites (NAA, $10 \mathrm{mM}$; creatine, $6 \mathrm{mM}$; Gln, $2 \mathrm{mM}$; Asp, $2 \mathrm{mM}$; and GABA, $2 \mathrm{mM}$ ) contributed negligible CEST effects to GluCEST signal (Supplementary Fig. 3).

For GluCEST imaging, three horizontal T2-weighted anatomical slice (thickness $=2 \mathrm{~mm}$ ) images were acquired. Of them, the second one that crosses through the dorsal hippocampus and PFC was selected as the target slice (Supplementary Fig. 4A). The hippocampus and PFC are two brain regions essential for advanced brain functions and compromised in some of mental disorders including anxiety, depression and schizophrenia ${ }^{23-25}$. The mean $\mathrm{B}_{0}$ shift (Supplementary Fig. $4 \mathrm{~B},{ }^{<} 0.5 \mathrm{ppm}$ ) and the $\mathrm{B}_{1} / \mathrm{B}_{1 \text { ref }}$ ratio (Supplementary Fig. $4 \mathrm{C}$, about 0.5 ) measured on this slice showed the rather good homogeneity of $\mathrm{B}_{0}$ and $\mathrm{B}_{1}$ fields. Relative to the GluCEST map of Control group (Fig. Supplementary 4D), the MSEW rats (Supplementary Fig. 4E) exhibited GluCEST contrast increases across the whole slice, reflecting a global increase in Glu concentration. The Z-spectra and MTRasym (asymmetrical magnetization transfer ratio) curves also indicate higher GluCEST contrast in the brain of MSEW rat compared to Control group (Supplementary Fig. 4F).

Following the global GluCEST scanning, regional analysis of GluCEST contrast was done. The regions of interest (ROIs) were PFC (Fig. 2A,B) and dorsal hippocampus (Fig. 2D,E) which were manually drawn based on the T2-weighted reference image of each rat. The corresponding Z-spectra and MTRasym curves of these two ROIs (Fig. 2C,F, respectively) were obtained. The GluCEST maps showed higher GluCEST contrasts in both PFC and hippocampus of MSEW rats (Fig. 2B,E, respectively) compared to those in the Control group (Fig. 2A,D, respectively). This observation was confirmed by Z-spectra and MTRasym curves (Fig. 2C,F). The summarized mean GluCEST contrasts in PFC and hippocampus showed higher GluCEST contrasts in both PFC and hippocampus of MSEW group compared to the Control group (Fig. 2G), suggesting that MSEW increased Glu levels in the two brain regions. 
A

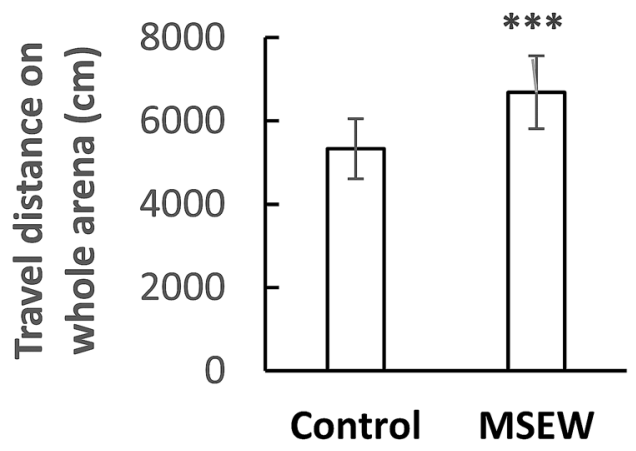

C

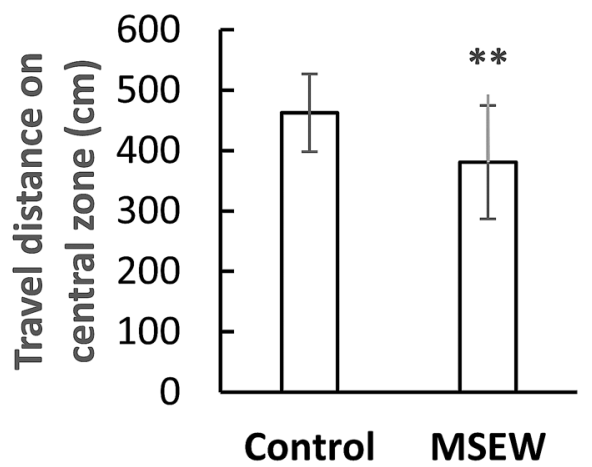

B

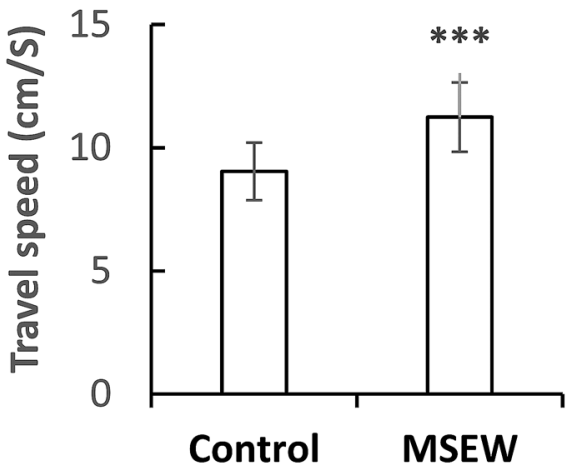

Figure 1. MSEW increased locomotor activity and anxiety level of rats. (A) MSEW rats moved a longer distance on whole arena of the open field compared to Control group. (B) MSEW rats moved at a higher velocity on the open field compared to Control group. (C) MSEW rats moved a shorter distance on the central zone of the open field compared to Control group. (D) MSEW rats spent less time on the central zone of the open field compared to Control group. Data are expressed as mean \pm SD. $N=15$ /group. Comparisons were made between Control and MSEW groups. ${ }^{* *} p<0.01 ;{ }^{* * *} p<0.001$.

MSEW impaired the integrity of neuron-astrocyte and axon-myelin entities in rat brain: detected by ${ }^{1} \mathrm{H}-\mathrm{MRS}$ and DTI. Increased Glu levels in PFC and dorsal hippocampus of MSEW rats suggest a possibility that the neuron-astrocyte entity was damaged by the MSEW paradigm. To confirm this suggestion, we performed ${ }^{1} \mathrm{H}$-MRS scanning with rats following GluCEST scanning. The two volumes of interest (VOIs) were located on the right side as shown in Fig. 3 (the inserts). Two representative ${ }^{1} \mathrm{H}$-MRS spectra obtained at frontal cortex were overlapped as shown in Fig. 3A (blue-Control; red- MSEW). The quantitative data of brain metabolites in this VOI of the two groups are shown in Fig. 3B indicating significantly higher levels of Glu, Glx (Glu + Gln), and NAA, but lower level of GABA, compared to Control group. Two representative ${ }^{1} \mathrm{H}$-MRS spectra obtained at the dorsal hippocampus were overlapped as shown in Fig. 3C (blue-Control; redMSEW). The quantitative data of brain metabolites in this VOI of the two groups are shown in Fig. 3D indicating significantly higher levels of Glu, NAA, NAAG, and Tau, compared to Control group.

In addition to NAA being used as the neurochemical correlate of axon-myelin entity as mentioned above, fractional anisotropy (FA) in DTI is commonly accepted as a biophysical index of myelin sheath integrity in the brain. It is a scalar value of the degree of directional diffusion within a voxel and linked to axon diameter, membrane permeability, and myelination. It is highly sensitive to microstructural changes within the location measured $^{26}$. Therefore, we performed DTI with the rats to look at if MSEW changed FA values in the rat brain. It was shown that FA values in frontal cortex, dorsal hippocampus, corpus callosum, and caudate putamen of MSEW rats were significantly lower than those in Control group (Fig. 4).

MSEW disrupted glutamate-glutamine-GABA cycle in PFC of rats: the molecular underpinnings of impaired neuron-astrocyte integrity. To reveal the molecular underpinnings of impaired neuron-astrocyte entity shown by non-invasive neuroimaging methods, we measured the protein levels of ATP- $\alpha$, GLT-1 (glial glutamate transporter 1), GLAST (the glutamate-aspartate transporter), PAG (phosphateactivated glutaminase), GAD65 (glutamate decarboxylase), and GS (glutamine synthetase) in the tissue sample of PFC. All these proteins involve in maintaining glutamatergic and GABAergic neurotransmission at opti- 

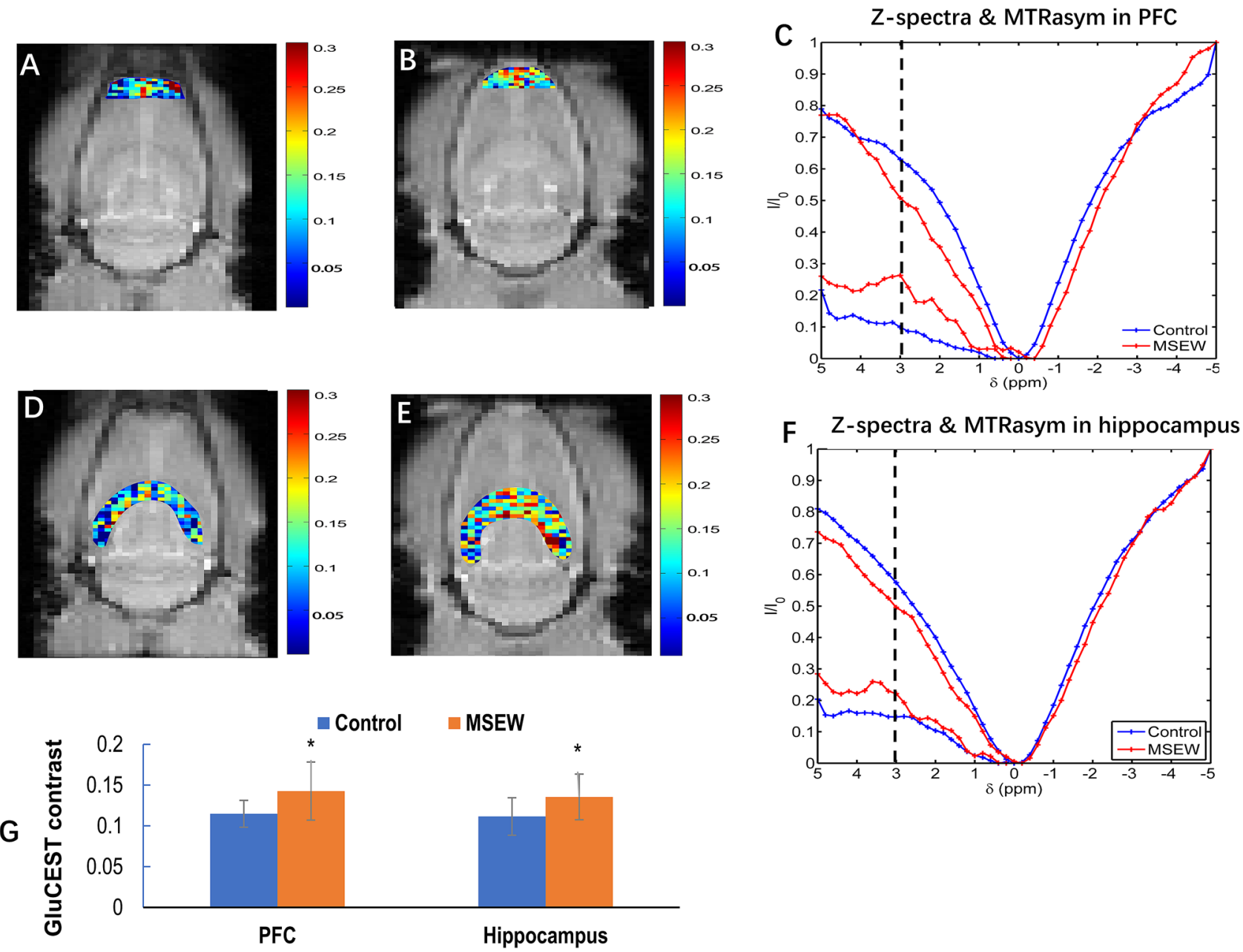

Figure 2. MSEW increased GluCEST contrast in PFC and dorsal hippocampus of rats. (A,B) The GluCEST map in PFC of Control and MSEW rats, respectively. (C) Corresponding z-spectra and MTRasym curves acquired at PFC of Control and MSEW rats. (D,E) The GluCEST map in dorsal hippocampus of Control and MSEW rats, respectively. (F) Corresponding z-spectra and MTRasym curves acquired at dorsal hippocampus of Control and MSEW rats. (G) Bar chart comparing the GluCEST contrasts in PFC and dorsal hippocampus of Control and MSEW rats. Data are expressed as mean \pm SD. $N=13-14$ /group in two batches. Comparisons were made between Control and MSEW groups. ${ }^{*} p<0.05$.

mal levels as reviewed in literature ${ }^{27-29}$. Specifically, after a glutamatergic transmission, astrocytes take up most of extracellular Glu via GLT1 and GLAST. And this Glu uptake process activates ATP- $\alpha$ to maintain the $\mathrm{Na}^{+}$ homeostasis ${ }^{30}$. Via this mechanism, astrocytes are able to preserve the extracellular concentration of Glu at low levels thus preventing excitotoxicity and keeping proper receptor-mediated functions ${ }^{28,31}$. Once taken up into the astrocytes, Glu is rapidly converted to Gln by GS which is largely restricted to this cell type. Then Gln is released into extracellular space where it can be taken up by glutamatergic and GABAergic neurons. In glutamatergic neurons Gln is converted to Glu by the neuron-specific enzyme PAG ${ }^{27}$. In GABAergic neurons, the reaction can go further and produce GABA in the presence of GAD ${ }^{29}$. Western blot analysis results showed lower levels of ATP- $\alpha$ and GLT-1, but higher levels of GAD65 and GS in PFC of MSEW rats compared to Control group, while no changes were found in levels of GLAST and PAG (Fig. 5A,B).

MSEW inhibited OLs maturation and myelination in PFC of rats: the cellular and molecular underpinnings of impaired neuron-oligodendrocyte integrity. To explore the cellular and molecular underpinnings of the MSEW-induced NAA increase and FA decrease in rat brain, we measured the protein levels of $\mathrm{N}$-acetyltransferase 8-like (NAT8L) and aspartoacylase (ASPA) in tissue sample of PFC. NAT8L catalyzes the synthesis of NAA from acetyl-CoA and aspartate in neurons whereas ASPA works for the hydrolyzation of NAA into aspartate and acetate in OLs ${ }^{32}$. Western-blot analysis showed lower levels of ASPA and NAT8L in PFC of MSEW rats compared to the Control group (Fig. 6), suggesting that the MSEW experience led to deficits in the expression of these two enzymes in OLs and neurons, respectively.

Concurrent NAA increase and ASPA decrease in PFC of MSEW rats suggest that the MSEW-induced functional deficit in OLs may happen in mature OLs or at advanced stages during OL development as ASPA activity increases markedly after PD7 and coincides with the time course for the onset of myelination in the rat brain ${ }^{33}$. 

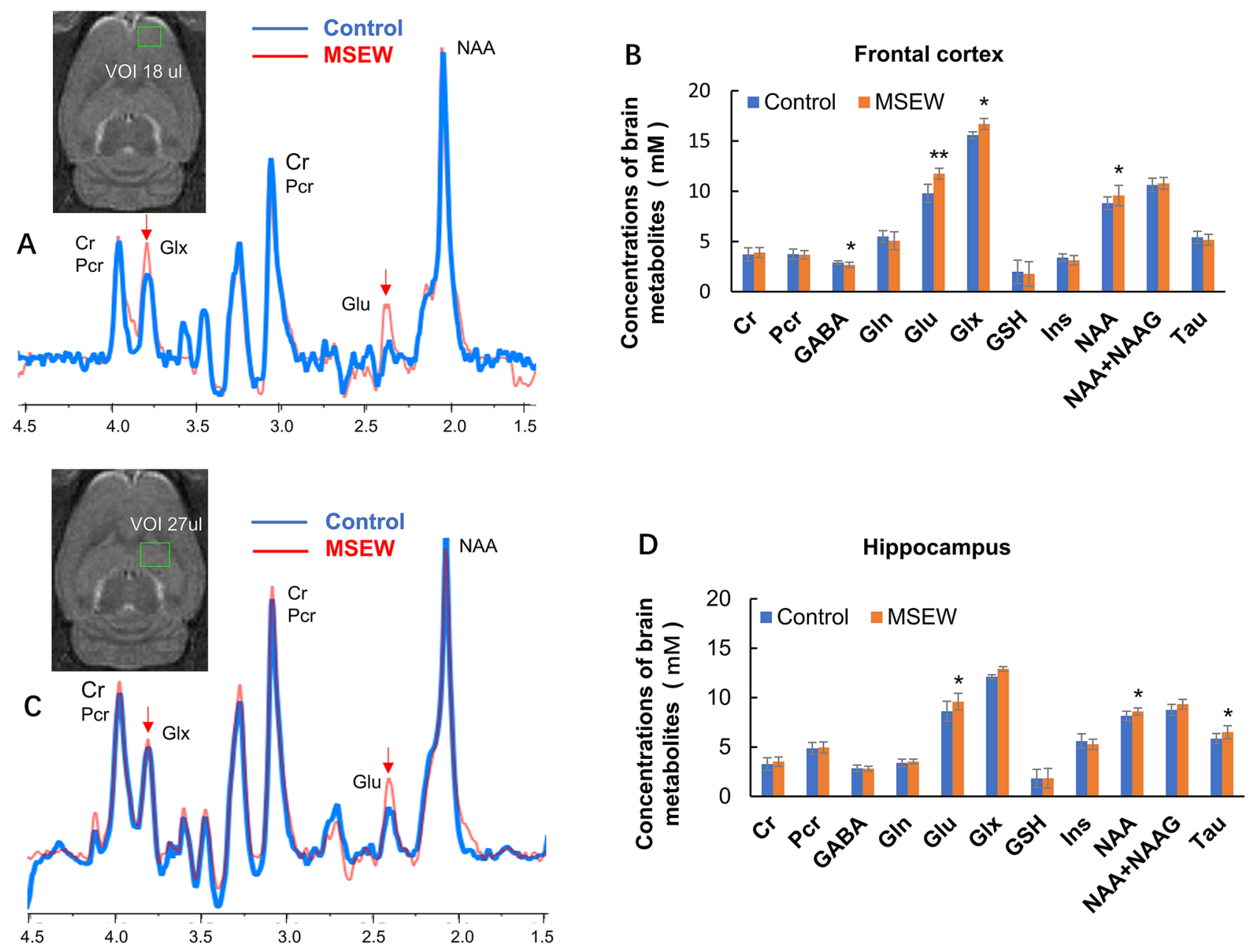

Figure 3. MSEW changed the neurochemical correlates of neuron-astrocyte integrity in PFC and dorsal hippocampus of the rat. (A) The overlapped two ${ }^{1} \mathrm{H}-\mathrm{MRS}$ spectra obtained at PFC of two rats in Control and MSEW groups, respectively (control: blue; MSEW: red). (B) Bar chart comparing levels of the brain metabolites in PFC of rats in the two groups. (C) The overlapped two ${ }^{1} \mathrm{H}-\mathrm{MRS}$ spectra obtained at dorsal hippocampus of two rats in Control and MSEW groups, respectively (control: blue; MSEW: red). (D) Bar chart comparing levels of the brain metabolites in dorsal hippocampus of rats in the two groups. Data are expressed as mean \pm SD. $\mathrm{N}=13-14$ /group in two batches. Comparisons were made between Control and MSEW groups. ${ }^{\star} p<0.05$; ${ }^{* *} p<0.01$.

To demonstrate this suggestion, we evaluated the mRNA expression of the transcription factors Olig2 and MRF (myelin-gene regulatory factor), as well as those of the myelin genes $M b p$ and Plp1 in PFC of rats. Olig2 is an OL-lineage determination factor necessary for the OL lineage specification within the $\mathrm{CNS}^{34,35}$; whereas MRF is necessary for the generation of mature, myelin gene expressing OLs in the brain ${ }^{36}$. RT-PCR analysis showed lower mRNA levels of $M R F, M b p$, and Plp1 in PFC of MSEW rats compared to Control group, but no difference was found in mRNA level of Olig2 between the two groups (Fig. 7A). Western-blot analysis showed lower levels of MBP protein (both MBP17 and MBP21 isoforms) in PFC of MSEW rats compared to Control group, but no difference was found in PLP1 levels between the two groups (Fig. 7B,C). Moreover, we performed immunohistochemical staining using the antibody to MBP. The MBP immunoreactivity in the sub-regions of cingulate cortex (Cg1), prelimbic cortex (PrL), lateral orbital cortex (LO), and ventral orbital cortex (VO) of PFC was measured. The results indicate that MSEW rats had lower MBP immunoreactivity in PrL and VO compared to those in Control group, but that in $\mathrm{Cg} 1$ and $\mathrm{LO}$ was comparable between the two groups (Fig. 8A-C).

\section{Discussion}

MSEW rats moved longer distance at a higher velocity on the whole arena of open field compared to those in the Control group. This is in line with a previous study showing that MSEW mice moved faster and walked a longer distance on the whole arena relative to those in Control group ${ }^{13}$. Moreover, MSEW rats, like MSEW mice ${ }^{13}$, spent less time at the central zone of the open field than controls, indicating a higher anxiety level. Increased anxiety-like behavior and locomotor activity were also seen in MSEW adult male mice in a recent study ${ }^{37}$. Taken together, the results of present study and previous ones support the notion that MSEW paradigm is a reliable model in mimicking early life neglect and abuse in humans. 
A

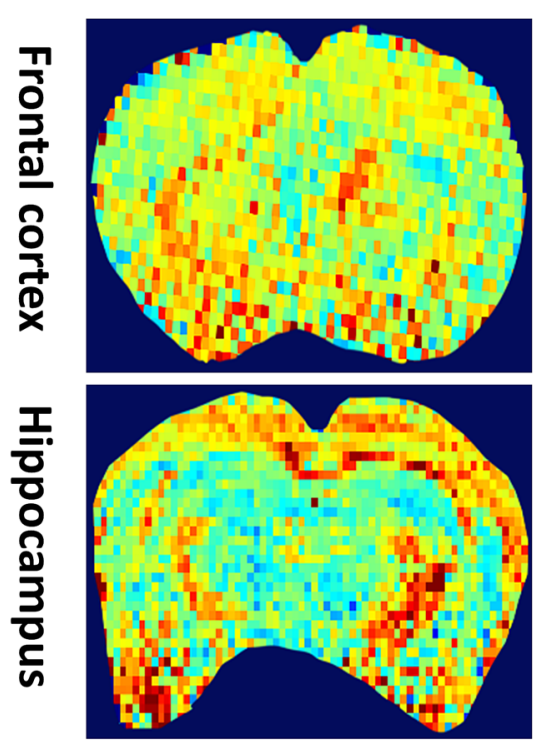

MSEW
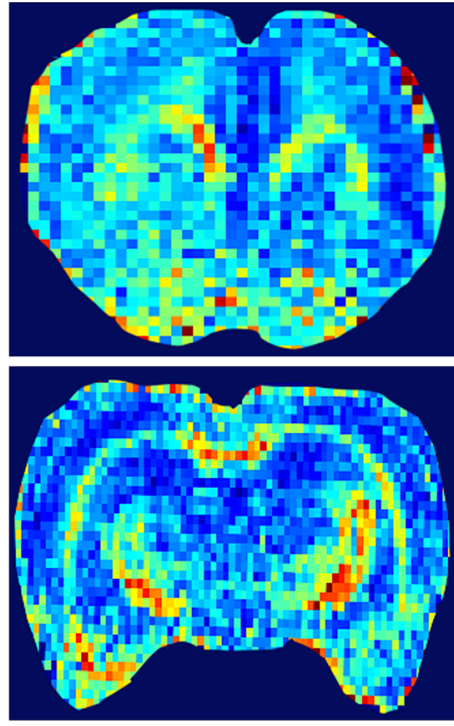

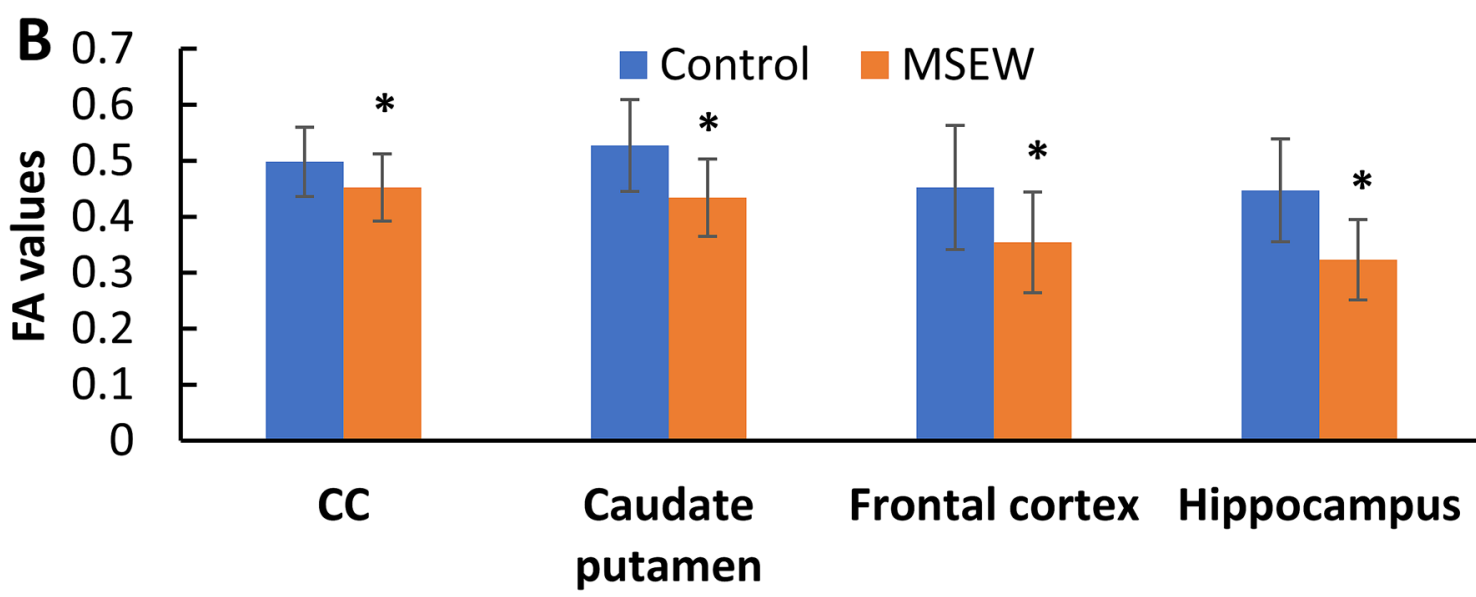

Figure 4. MSEW damaged the white matter integrity of rat brain. (A) Representative DTI maps from Control and MSEW rats showing MSEW-induced FA decreases in CC, caudate putamen, frontal cortex, and hippocampus. (B) Bar chart comparing FA values in all the measured brain regions of the two groups. Data are expressed as mean \pm SD. $\mathrm{N}=11-18$ /group in two batches. ${ }^{*} p<0.05$.

The GluCEST maps and regional analysis of GluCEST exhibited increased GluCEST contrast in PFC and dorsal hippocampus of MSEW rats relative to Control group, suggesting increased Glu concentrations in the two brain regions. In support of this suggestion, a previous study reported a significant increase in GluCEST contrast in rats $24 \mathrm{~h}$ after administration of modafinil, a drug known to increase Glu levels in the brain ${ }^{38}$. In the other studies, restraint and swimming stress increased extracellular levels of endogenous excitatory amino acids, aspartate and Glu ${ }^{39,40}$, and the stress-induced release of Glu is more pronounced in PFC of the rat as compared to other regions tested ${ }^{40,41}$. All these point to a conclusion that GluCEST is a reliable non-invasive imaging method able to measure brain Glu levels in living subjects and has specific relevance to clinical research on patients with mental disorders thereof.

${ }^{1} \mathrm{H}$-MRS revealed higher levels of Glu, but lower GABA in PFC of MSEW rats relative to Control group. According to our neuron-glia integrity theory, changes in levels of Glu, Gln, and/or GABA suggest a disrupted Glu-Gln-GABA cycle in the neuron-astrocyte entity in the brain ${ }^{9}$. In support of this suggestion, we revealed the molecular changes underlying the MSEW-induced damage to neuron-astrocyte entity as described below. First, MSEW paradigm induced concurrent decreases in levels of GLT-1 and ATP- $\alpha$ in PFC of rats. These two proteins were reported to co-localize within the final processes of astrocytes in two previous studies ${ }^{42,43}$ and their interactions were demonstrated in the other studies ${ }^{4-47}$. In light of these recent advances, it is highly likely that 
A
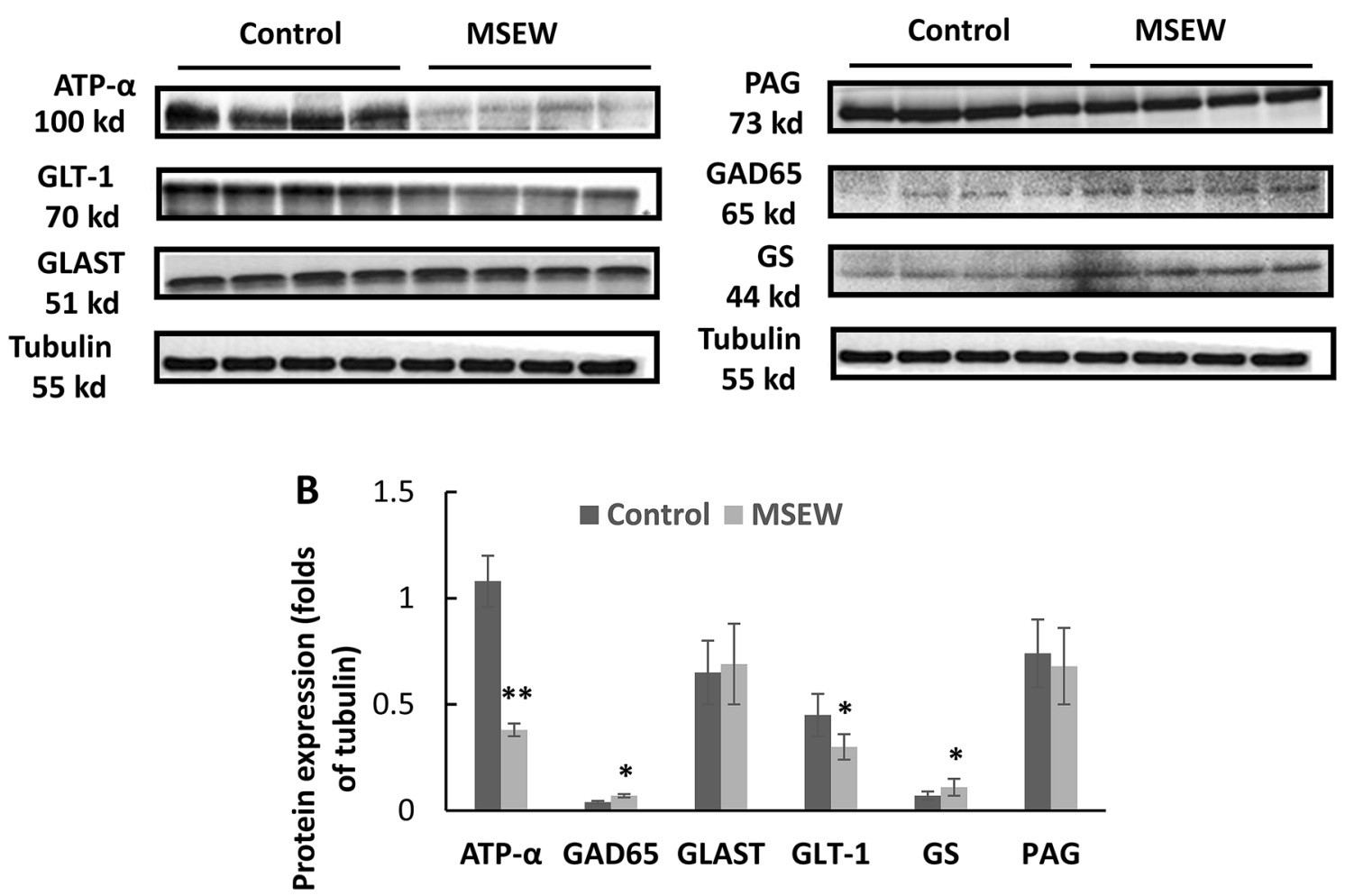

Figure 5. Molecular underpinnings of impaired neuron-astrocyte integrity in MSEW rats. (A) Representative western-blot images of the target proteins (including ATP- $\alpha$, GLT-1, GLAST, PAG, GAD65, and GS) in PFC of rats, along with that of $\alpha$-tubulin as the reference. (B) Bar chart comparing levels of all the measured proteins of the two groups. Data are expressed as mean $\pm \mathrm{SD} . \mathrm{N}=8$ /group. Comparisons were made between Control and MSEW groups. ${ }^{*} p<0.05 ;{ }^{*} p<0.01$. The samples derived from the same experiment and gels/blots were processed in parallel. The original images of Western blot for this figure are presented in supplementary materials.
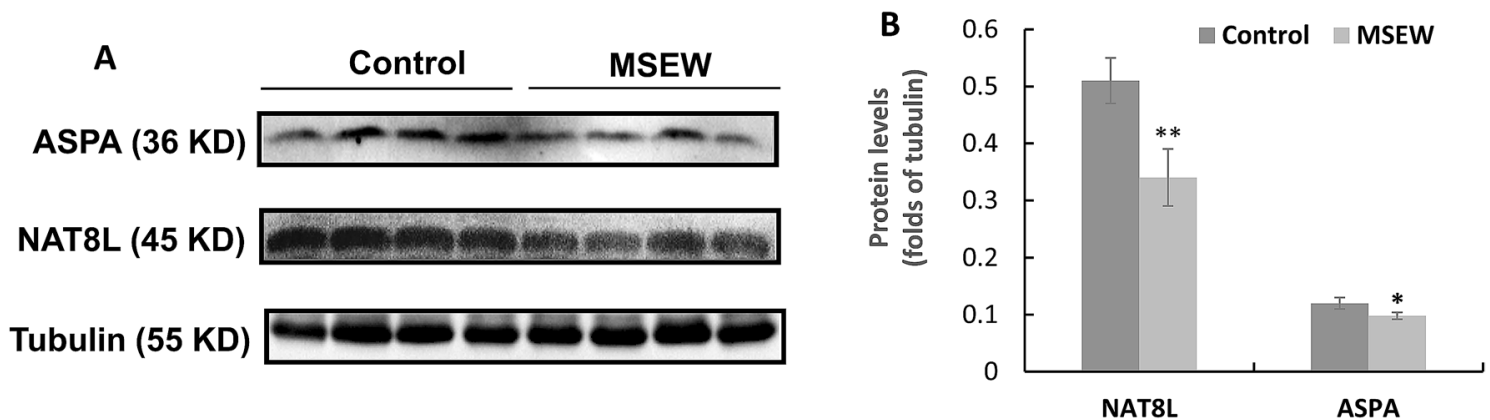

Figure 6. MSEW decreased levels of ASPA and NAT8L in PFC of the rat. (A) Representative western-blot images of ASPA and NAT8L in PFC of rats, along with that of $\alpha$-tubulin as reference. (B) The bar chart comparing levels of ASPA and NAT8L in PFC of rats in Control and MSEW groups. Data are expressed as mean \pm SD. $N=8$ /group. Comparisons were made between Control and MSEW groups. ${ }^{*} p<0.05$. The samples derived from the same experiment and gels/blots were processed in parallel. The original images of Western blot for this figure are presented in supplementary materials.

concurrent decreases in levels of GLT-1 and ATP- $\alpha$ slowed down the uptake of Glu and the transport of it into astrocytes thus leading to Glu increase in the synaptic cleft and an initial decrease of Glu in astrocytes. Under this circumstance, the astrocytes in MSEW rats must increase de novo synthesis of Glu thus leading to a subsequent increase of Glu as revealed by GluCEST and ${ }^{1} \mathrm{H}-\mathrm{MRS}$. Second, the conversion of Glu to Gln in astrocytes was sped up as evidenced by the upregulation of GS in the MSEW rats. Third, the protein levels of PAG, the neuronspecific enzyme responsible for the conversion of Gln to Glu, were comparable between the Control and MSEW groups, indicating that the conversion of Gln into Glu in glutamatergic neurons occurred normally, which may 
A
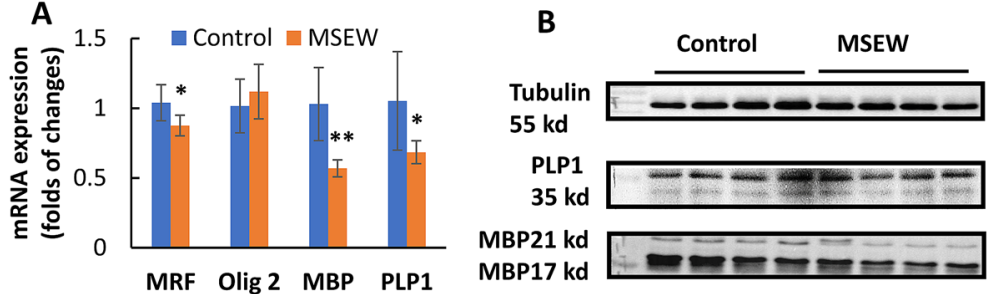

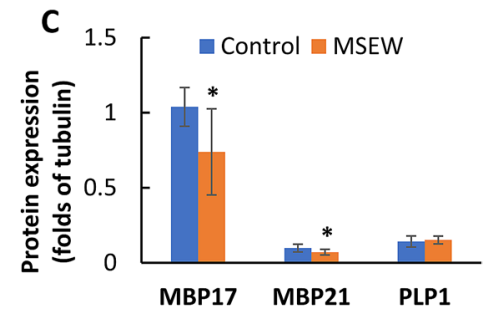

Figure 7. MSEW inhibited OLs maturation and myelination in PFC of rat brain. (A) The summarized data showing that MSEW decreases mRNA levels of MRF, MBP, and PLP1 genes in PFC of rats, but has no effect on that of Olig2 gene. (B) Representative western-blot images showing the immunoreactive bands of MBP (including MBP17 and MBP21 isoforms) and PLP1 proteins in PFC of rats. The samples derived from the same experiment and gels/blots were processed in parallel. The original images of Western blot for this figure are presented in supplementary materials. (C) Bar chart comparing levels of MBP (including MBP17 and MBP21 isoforms) and PLP1 proteins in PFC of Control and MSEW groups.
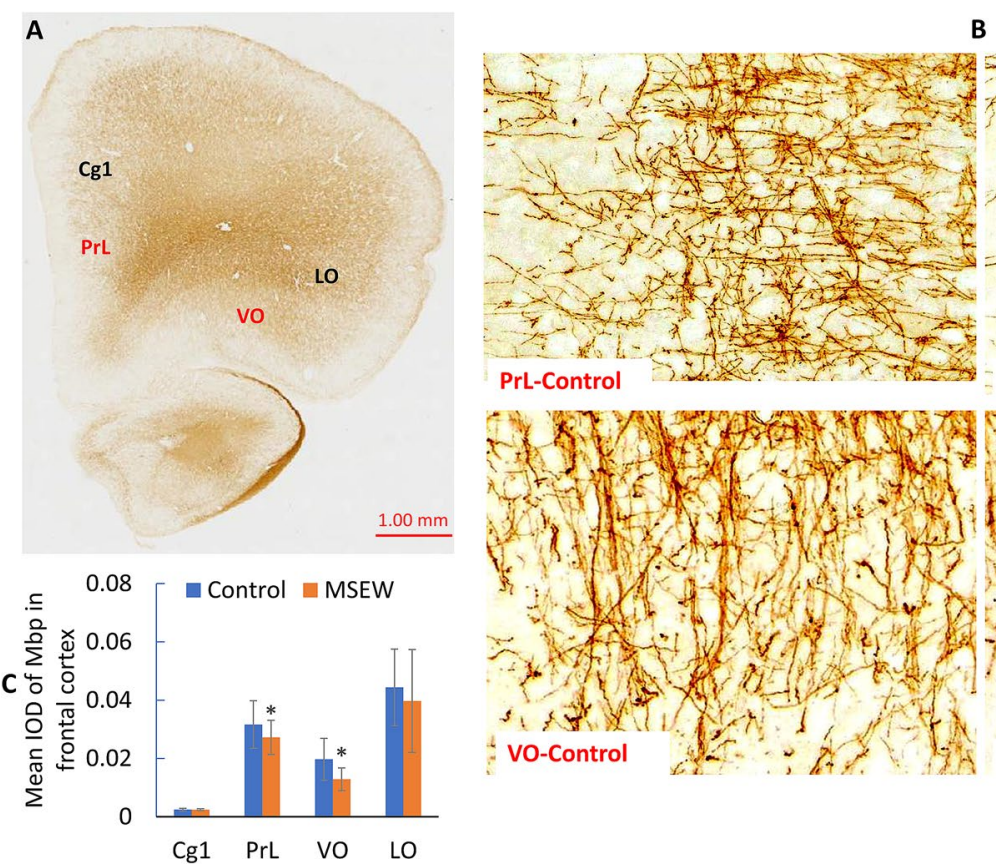

B

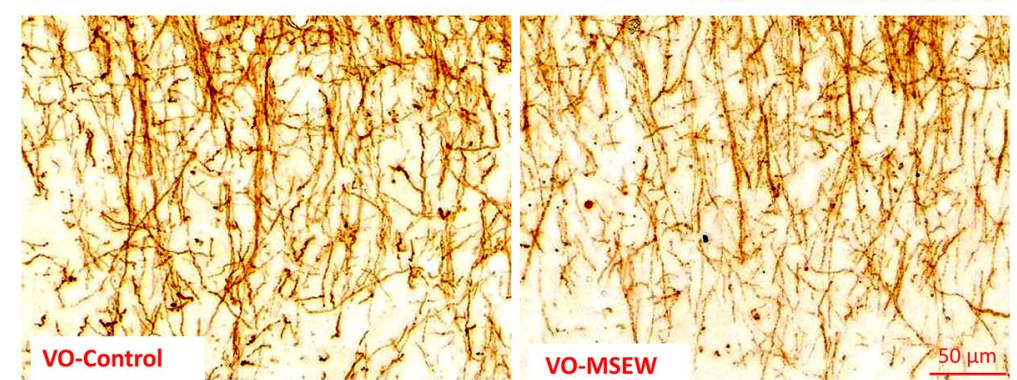

Figure 8. MSEW decreased MBP immunoreactivity in the sub-regions PrL and VO of PFC of rat brain. (A) A representative immunochemical staining microphotograph taken at a lower magnification showing the subregions of Cg1, LO, PrL, and VO of PFC. (B) Representative microphotographs taken at higher magnifications showing the MBP immunohistochemical staining in PrL and VO in PFC of rats. (C) Bar chart comparing MBP immunoreactivity in the sub-regions Cg1, LO, PrL, and VO in PFC of Control and MSEW rats. Data are expressed as mean \pm SD. $N=6-8$ /group. Comparisons were made between Control and MSEW groups. ${ }^{*} p<0.05,{ }^{* *} p<0.01$. Cgl cingulate cortex, $L O$ lateral orbital cortex, $\operatorname{PrL}$ prelimbic cortex, $V O$ ventral orbital cortex.

account for the comparable levels of Gln in MSEW and Control groups. Forth, MSEW rats showed higher level of GAD65, the enzyme catalyzing the conversion of Glu into GABA in GABAergic neurons, but GABA level in PFC of MSEW rats was lower than Control group, suggesting that GAD65 increase in GABAergic neurons was not competent enough to maintain GABA at normal levels.

Interestingly, Glu increase was not accompanied by GABA decrease in the dorsal hippocampus of MSEW rats. Although this study did not address why PFC and dorsal hippocampus showed different responses to MSEW in terms of GABA levels, this phenomenon is in line with previous preclinical findings. For example, chronic unpredictable stress for $16 \mathrm{~d}$ caused a selective reduction of somatostatin (SST)/calbindin interneurons in the PFC, but no change in hippocampus ${ }^{48,49}$. In a recent study, the numbers of parvalbumin (PV)- and SSTexpressing interneurons in medial PFC (mPFC) of BRINP1-KO mice were, respectively, $50 \%$ and $20 \%$ fewer than corresponding neurons in $\mathrm{mPFC}$ of wild-type mice, but this change was not found in the hippocampus ${ }^{50}$. The authors attributed hyperactive locomotive activity of BRINP1-KO mice to the decreased GABAergic neurons 
in $\mathrm{mPFC}^{51}$. Similarly, the decreased GABA in PFC, but not in dorsal hippocampus, of MSEW rats may explain increased locomotor activity of them in open field test.

The pathologically elevated NAA in MSEW rats is indicative of impaired axon-myelin/neuron-OL entity as NAA level in the brain depends on the normal functions of NAA synthase in neurons and aspartoacylase in OLs. Higher levels of NAA indicate the degradation of NAA into L-aspartate and acetate was inhibited in OLs thus leading to deficit in acetate, a building block required for myelin lipid synthesis in OLs ${ }^{9,52}$. Further evidence associating higher NAA level to axon-myelin integrity impairment in the brain is the concurrent lower FA values in PFC and hippocampus of MSEW rats. As mentioned before, FA is commonly accepted as a biophysical index of myelin sheath integrity in the brain. Altered FA values have been correlated to changes in myelination and axonal membrane integrity ${ }^{53}$. In line with our interpretation, previous studies showed: (1) MSEW significantly lowered FA values in medial septum and anterior commissure of mice ${ }^{54}$; and (2) pubertal verbal stress in humans was associated with adult decreased FA values in several brain regions ${ }^{55}$. More compelling evidence came from a recent study of our team, in which intermittent social defeat stress led to temporary decreases in the levels of total creatines $(\mathrm{Cr}+\mathrm{pCr})$ and $\mathrm{Glx}(\mathrm{Glu}+\mathrm{Gln})$ seen in the first ${ }^{1} \mathrm{H}-\mathrm{MRS}$, and a delayed increase of NAA levels shown in the second ${ }^{1} \mathrm{H}$-MRS. These alterations were accompanied by a persistent reduction of MBP immunoreactivity in $\mathrm{mPFC}$ of Balb/c mice. While attributing decreased levels of total creatines and Glx seen in the first ${ }^{1} \mathrm{H}$-MRS to the stress-induced damage to neuronal mitochondria, we noticed that the stress damaged OLs too as evidenced by decreased MBP immunoreactivity. After a 3-week recovery period, damage to neurons completely recovered as the only change in the second ${ }^{1} \mathrm{H}$-MRS was an increased NAA peak. Explicitly, this NAA increase is indicative of damaged mitochondria in OLs, where degradation of NAA into L-aspartate and acetate occurs as mentioned before. In other words, the stress-induced damage to OLs did not normalize during this 3-week recovery period. Indeed, MBP immunoreactivity remained at lower levels over the whole 3-week period ${ }^{56}$. These results suggest that brain neurons are more resistant to stress-induced mitochondrial damage relative to OLs. This suggestion has been elegantly tested true in a very recent study of our team, which showed that brain neurons are most sensitive but most resistant to mitochondria oxidative stress whereas mature OLs are sensitive and most vulnerable ${ }^{57}$. Relevantly, patients with Canavan's disease show NAA accumulation along with myelin defect due to the deficiency of ASPA ${ }^{58}$.

Like in the case of Canavan's disease, MSEW rats in the present study showed decreased ASPA levels in PFC, suggesting functional deficit of OLs. Moreover, MSEW decreased NAT8L levels in PFC, suggesting the synthesis of NAA from acetyl-CoA and aspartate in neurons was also impaired. Via these actions, MSEW led to hypomyelination in the rat brain as evidenced by lower levels of MBP protein in PFC detected by means of Western-blot and immunohistochemical staining. Of note, MBP immunoreactivity in PrL and VO subregions of PFC was significantly lower in MSEW rats compared to Control group. These two subregions are in the lower medial and orbital prefrontal cortices in rodents and typically considered to be related to human and monkey Brodmann area 25, a component of ventromedial prefrontal cortex, a major player in social and affective functions ${ }^{23,59}$. Moreover, MSEW rats showed significantly lower levels of MRF mRNA, but no change in Olig2 mRNA levels, suggesting that the MSEW procedure affected OL maturation, but had no effect on OL differentiation. All the aforementioned data have confirmed the validity of NAA being used as a neurochemical index of axon-myelin integrity and revealed the mechanism by which MSEW impaired the myelinating function of OLs thus damaged the axon-myelin integrity in rat brain.

\section{Conclusions}

In conclusion, MSEW disrupted neuron-glia integrity in PFC and dorsal hippocampus of rats as evaluated by non-invasive neuroimaging methods showing changes in levels of Glu, NAA, and GABA in the brain regions, as well as global decrease in FA value. Underlying the disrupted neuron-astrocyte integrity in MSEW rats, changes happened in levels of the proteins GLT-1, ATP- $\alpha$, GAD65 and GS involved in maintaining glutamatergic and GABAergic neurotransmission at optimal levels. Accounting for damaged axon-myelin integrity, MSEW lowered levels of ASPA and NAT8L, as well as MBP levels and MBP immunoreactivity in PFC. Along with these changes, MSEW increased locomotor activity and anxiety levels of rats. All the data substantiate the neuron-glia integrity theory and relate neuron-glia integrity impairment to emotional dysfunction in the rat.

\section{Methods}

Animals. Female S-D rats at gestational week 2 were purchased from the animal center of the Southern Medical University (Guangzhou, China) and housed in an air-conditioned room at Shantou University Medical College. The animals had free accesses to food and water in the room with controlled temperature in the range of $23 \pm 1{ }^{\circ} \mathrm{C}$ and a 12:12 $\mathrm{h}$ light cycle. The delivery day was defined as PD0. An even number up to ten pups (males) of each litter and their dam were culled to proceed to the next MSEW procedure or be used as controls. All animal handling and use were carried out in accordance with the guidelines set up by the Animal Care and Use Committee of Shantou University Medical College and approved by the committee.

The MSEW procedure. The maternal separation started on PD2, by removing a pup from his/her dam and placing the pup in a small paper box $(10 \times 9 \times 9 \mathrm{~cm})$ for $4 \mathrm{~h}$ during PDs $2-5$, and $6 \mathrm{~h}$ during PDs 6-16. During the separation periods, which started at the same time $(8: 00 \mathrm{am})$ every day, pups in cartons (one pup per carton) were kept at an infant incubator (YP-100; Ningbo David Medical Device Co., Ltd., Ningbo, China) which was kept well ventilated at a controlled temperature $\left(34^{\circ} \mathrm{C}\right.$ during PDs $2-5,32^{\circ} \mathrm{C}$ during PDs6-9, $30^{\circ} \mathrm{C}$ during PDs $10-14$, and $28^{\circ} \mathrm{C}$ during PDs $\left.15-16\right)$ and humidity (60\%). Before and after maternal separation, all pups in the MSEW group were brought back to the cage where their dam was living. Weaning occurred on PD 17 when a home-made soft diet (powdered rodent chow in tap water) was provided to the isolated pups. Starting on PD 22, 
the MSEW rats of a same litter were housed in group (10 pups/cage). The pups in Control group were raised by their dams under the standard laboratory condition as described before and weaned on PD 22. The body weight of all pups was weighed on PDs 7, 14, 21, and PD 30, respectively. During PDs 60-62, rats in the MSEW and Control groups were subjected to the open-field test followed by non-invasive neuroimaging scanning including DTI, GluCEST, and ${ }^{1} \mathrm{H}$-MRS. This experimental schedule did not allow blood sampling for cortisol evaluation of rats.

Open field test. Open field test was performed to measure the locomotor activity, exploratory behavior, and anxiety-related behaviors of rats. The wooden open field box $(100 \times 100 \times 60 \mathrm{~cm})$ was painted in black and sheltered by a blue drape in the behavioral test room, which was lighted with three fluorescents of 15 lumens. The experimental method has been described in a previous study ${ }^{60}$. Briefly, each individual rat was placed in the center of the open field box and allowed to move freely for $12 \mathrm{~min}$, of which the first two minutes were defined to be the adaptation period and the data of this period was not included for analysis. A video tracking system (EthoVision XT 9.0; Noldus Information Technology, Wageningen, Netherlands) was used to monitor the tested rat. For each test, the travel distances on the whole arena and its central zone (the central part of $50 \times 50 \mathrm{~cm}$ ), the travel speed, and time spent on the peripheral and central zones were recorded. The floor and inner walls of the box were cleaned with $70 \%$ ethanol after each test.

Phantom scanning of MRI. All MRI scanning with phantom and animal were performed using an animal MRI scanner (7 T, Agilent Technologies, Santa Clara, CA, USA) along with a $63 \mathrm{~mm}$ internal diameter standard proton transmission and reception volume coil. During MR imaging and spectroscopy measurements, animals were kept under anesthesia (1.5\% isoflurane in $1 \mathrm{~L} /$ min oxygen) and their body temperature maintained at $36-37^{\circ} \mathrm{C}$

A phantom was prepared as described in a previous study to optimize CEST MRI procedure ${ }^{61}$. Briefly, $1 \%$ agarose was added into a phosphate-buffered saline (PBS) solution in test tubes ( $5 \mathrm{~mm}$ in diameter) and heated by a microwave. The mixture was then immersed in a water bath at $45^{\circ} \mathrm{C}$. Glu (Sigma-Aldrich, St Louis, MO, USA) was added at the various concentrations of $0,3,6,9,12$, and $15 \mathrm{mM}$, and the $\mathrm{pH}$ was titrated to 7.0. The tubes were inserted into a phantom holder filled with $1 \%$ agarose gel to minimize susceptibility inhomogeneity. In addition, another phantom consisting of tubes with solutions of different metabolites at their physiological concentrations (Asp, $2 \mathrm{mM}$; Cr, $6 \mathrm{mM}$; GABA, $2 \mathrm{mM}$; Gln, $2 \mathrm{mM}$; Glu, $10 \mathrm{mM}$; NAA, $10 \mathrm{mM}$ ). All these chemicals were purchased from Sigma-Aldrich (St Louis, MO, USA) and dissolved in a 1\% agarose gel at the $\mathrm{pH}$ of 7.0.

Diffusion tensor imaging. Diffusion tensor imaging was performed for anesthetized individual rat placed in the prone position with the head fixed on a palate holder equipped with an adjustable nose cone. Respiration and body temperature were monitored using an MRI compatible small animal monitor system (SA Instruments, Inc., Stony Brook, NY, USA). The body temperature of rat was maintained at $37^{\circ} \mathrm{C}$ using a water-heated animal blanket. High resolution anatomical $\mathrm{T}_{2}$-weighted images were acquired with multi-slices multi-echoes (MSME) sequence $(\mathrm{TE} / \mathrm{TR}=40 / 3000 \mathrm{~ms}$, average $=1$, repetition $=1$, matrix $=256 \times 256$, slice thickness $=2.0 \mathrm{~mm})$ in coronal (10 slices), sagittal (8 slices) slices. 4 sub-regions in 2 sagittal slices were orientated and used for accurate delineation of the target regions. DTI scanning was carried out by using a fast spin echo (FSE) diffusionweighted sequence with the parameters of $\mathrm{TR}=2000 \mathrm{~ms}$, repetitions $=1$, average $=8$, data matrix $=192 \times 128$, FOV $=45 \times 45 \mathrm{~mm}$, slice $=8$, thickness $=1 \mathrm{~mm}$, gap $=0.2 \mathrm{~mm}, \mathrm{~b}=1000 \mathrm{~s} / \mathrm{mm}^{2}$. DTI data were processed using Diffusion Toolkit in Track Visual software according to a previous study ${ }^{62}$. The axes of the ellipsoid are described by a set of eigenvectors and eigenvalues $(\lambda \mathrm{i})$ to calculate FA values obtained in four ROIs including CC, caudate putamen, frontal cortex, and hippocampus in corresponding target slices. All these DTI measures were obtained and analyzed by AFNI (Analysis of Functional NeuroImages) software.

GluCEST imaging. GluCEST imaging was done as described above in DTI subsection. $\mathrm{T}_{2}$-weight images were acquired. The second one of the horizontal slices passing through the frontal cortex and dorsal hippocampus was selected for GluCEST image. A custom-programmed segmented RF spoiled gradient echo (GRE) pulse sequence was used. The sequence parameters ${ }^{\star *}$ were as follows: $\mathrm{FOV}=50 \mathrm{~mm} \times 50 \mathrm{~mm}$. TR $=17.58 \mathrm{~ms}$; $\mathrm{TE}=2.77 \mathrm{~ms}$, flip angle $=15^{\circ}$, average $=8$, matrix $=128 \times 64$, slice thickness $=2 \mathrm{~mm}$. CEST images were collected using a $1 \mathrm{~s}$ saturation pulse at $\mathrm{B} 1$ of $250 \mathrm{~Hz}(5.9 \mu \mathrm{T})$ at multiple frequencies in the range -5 to $+5 \mathrm{ppm}$, with a step size of $0.2 \mathrm{ppm}$. The scan time was $7 \mathrm{~m} 57 \mathrm{~s}$ for each animal. Control images $\left(\mathrm{S}_{0}\right)$ were obtained at an offset of $300 \mathrm{ppm}$ for normalization, and GluCEST was measured at $3 \mathrm{ppm}$.

For MTR mapping, the images at $20 \mathrm{ppm}$ and $100 \mathrm{ppm}$ were collected on the same brain slice with a saturation power of $250 \mathrm{~Hz}$ and a saturation duration of $1 \mathrm{~s}$. An image at $100 \mathrm{ppm}$ was considered as the magnetization off image.

All GluCEST image processing and data analysis were performed using programs employed in previous studies $^{15}$. CEST images were corrected by B0 and B1 map and z-spectra were obtained from the normalized images for the ROI outlined. CEST contrast was calculated according to the Equation:

$$
\text { GluCEST }_{\text {asym }}=\frac{\mathrm{S}_{-3 \mathrm{ppm}}-\mathrm{S}_{+3 \mathrm{ppm}}}{\mathrm{S}_{0}}
$$

where $S_{ \pm 3 \text { ppm }}$ was the magnetization obtained with saturation at a ' + ' or '-' 3 ppm offset from the water resonance, in which $+3 \mathrm{ppm}$ was consider as frequency offset of Glu for chemical exchange and saturation transfer. $\mathrm{S}_{0}$ is the magnetization with a saturation pulse applied at $100 \mathrm{ppm}$, as a control image for data normalization. 


\begin{tabular}{|l|l|l|l|}
\hline Primer & Primer ID & Sequences & Genbank Acc. \\
\hline \multirow{2}{*}{ MBP } & RA051535-F & CCCATTGGTGCACACTAACCTC & \multirow{2}{*}{ NM_001025291.1 } \\
\cline { 2 - 4 } & RA051535-R & CGACTTGATTCAGCGACAGGA & \\
\hline \multirow{2}{*}{ PLP1 } & RA050858-F & AACTGCCAGCTGTTTAGTTGGTG & \multirow{2}{*}{ NM_030990.2 } \\
\cline { 2 - 4 } & RA050858-R & AGGTTTCCAATTCTGTCTGTCCTC & \\
\hline \multirow{2}{*}{ MRF } & RA054567-F & GTAAGGGCCACAGGCTGGAA & \multirow{2}{*}{ NM_001170487.1 } \\
\cline { 2 - 4 } & RA054567-R & CCAGTGGCTATAGGAACAGCAGAA & \\
\hline \multirow{2}{*}{ Olig 2 } & RA048959-F & ACCGAAGTAGTGAGAGCACTTGGAG & \multirow{2}{*}{ NM_001100557.1 } \\
\cline { 2 - 4 } & RA048959-R & ATGGATGTACCCGCGTGTTG & \\
\hline
\end{tabular}

Table 1. Primer sequences used for real-time RT-PCR.

MTR maps were also computed using Equation:

$$
\mathrm{MTR}_{\mathrm{asym}}=\frac{\mathrm{S}_{0}-\mathrm{S}_{+20 \mathrm{ppm}}}{\mathrm{S}_{0}}
$$

where $S_{+20 p p m}$ was the magnetization of macromolecules acquired with saturation pulse at $+20 \mathrm{ppm}$.

Proton MRS. Proton MRS was done as described in GluCEST subsection. A single voxel spectrum was acquired with point resolved spectroscopy (PRESS) using a vendor (Varian) provided pulse sequence. The parameters were as follows: $\mathrm{TR}=3000 \mathrm{~ms}, \mathrm{TE} 1=7.61 \mathrm{~ms}, \mathrm{TE} 2=6.01 \mathrm{~ms}$, TE total $=13.62 \mathrm{~ms}$, average $=12$, dummy scan $=2$, real points $=4095$, spectral width $=4005 \mathrm{~Hz}$, slice thickness $=2 \mathrm{~mm}$, voxel size in hippocampus $=3.0 \times 3.0 \times 3.0 \mathrm{~mm}$ and in frontal cortex $=3.0 \times 3.0 \times 2.0 \mathrm{~mm}$. The scan time was $21 \mathrm{~min} .3 \mathrm{D}$ voxel shimming was performed to obtain localized water line width values of lower than $12 \mathrm{~Hz}$. Water suppression was performed by the variable pulse power and optimized relaxation delays method (VAPOR). An unsuppressed water spectrum was also acquired using the same parameters for normalization. Both water-suppressed and water reference spectra were analyzed by LC-model. The metabolites were quantified under the condition of CramérRao lower bound (CRLB) $10 \%$ in experiments except GABA $<20 \%$. MRS spectra was fitted by MestReNova software.

RT-PCR analysis. Tissue samples were lysed using Trizol and chloroform. The extracted RNA was quantified by microplate reader (Tecan, M1000 Pro, China). Then, the RNA sample was diluted into $140 \mathrm{ng} / \mu \mathrm{L}$ and purified with the Primescript RT regent kit (Takara, RR047). cDNA was generated using SYBR Premix Ex Taq II kit (Takara, RR820A). Quantitative real-time PCR was run on the Applied Biosystems7500 Real Time PCR System (Applied Biosystems, Foster City, CA, USA) by following the 2 steps real time RT-PCR procedure and using primers listed in Table 1. Each CT value acquired from quantitative RT-PCR was calculated as average of triplicate samples from one rat (8 in each group). GADPH gene was used as the reference gene. Relative gene expression levels were analyzed using the $2^{\wedge}(-\Delta \Delta \mathrm{CT})$ method.

Western-blot analysis. The PFC samples of rats were homogenized, and proteins were extracted using a Tris-EDTA lysis buffer (1\% Triton X-100, 10\% glycerol, 20 mM Tris, pH 7.5, 1 mM EDTA) containing freshly added protease inhibitor cocktail (Sigma-Aldrich, St. Louis, MO, USA). After protein determination using a BCA kit, sodium dodecyl sulfate-polyacrylamide gel electrophoresis and Western blotting were performed. The antibody against $\beta$-tubulin (1:1000) (Sigma-Aldrich, St. Louis, MO, USA) was used as an internal control for the concentration of protein loaded, while the primary antibodies against ATP- $\alpha$ (1:1000), GLT-1 (1:5000), GLAST (1:2000), GAD65 (1:1000), GS (1:500), or PAG (1:1000) were used to detect the corresponding target proteins. After incubation in a secondary antibody solution (anti-rabbit antibody, 1:1000), the immunoreactive bands were visualized using an ECL detection kit (Amersham Biosciences, Buckinghamshire, UK). Image Lab software version 5.0 (Bio-Rad) was used for image acquisition and densitometric analysis of the immunoreactive bands. The data were expressed as folds of $\alpha$-tubulin band in a corresponding lane.

Immunohistochemical staining. Immunohistochemical staining was performed as described previously ${ }^{63}$. The brain sections (20 $\mu$ m thickness) were blocked with $5 \%$ goat serum (GeneTex, Alton PkwyIrvine, CA) in $0.01 \mathrm{M}$ PBS containing Triton X-100 (0.1\%) for $1 \mathrm{~h}$ at room temperature, followed by the incubation with the primary antibody against MBP (1:200; Abcam, Cambridge, UK) overnight at $4{ }^{\circ} \mathrm{C}$. After rinsed three times with PBS, the sections were incubated with HRP-conjugated secondary antibody (goat anti-rabbit IgG, 1:1000; Beyotime Biotechnology, Shanghai, China) at $37{ }^{\circ} \mathrm{C}$ for $1.5 \mathrm{~h}$ at room temperature. After rinsed in PBST, the sections were visualized by adding diamino-benzidine solution as recommended by the manufacturer (Zhongshan Gold Bridge Biology Company, Beijing, China). After rinsed in PBS, the sections were mounted onto the glass slides, dehydrated in gradient ethyl alcohol and cleared in xylene. After mounted by neutral balsam, immunohistochemical staining was observed and recorded with a Zeiss microscope (Zeiss instruments Inc., Germany) and analyzed with the Image-Pro-Plus 6.0 software (Media Cybernetics, Rockville, Maryland). 
The negative control staining was done following the same procedure except for the deletion of the primary antibody. No positive MBP immunoreactive staining was seen on the negative control sections.

Analysis of immunohistochemical staining. As described previously ${ }^{63}$, the quantitative analysis of immunohistochemical staining was performed with the sub-regions of Cg1, LO, PrL, and VO of PFC as ROIs. For each ROI, three sections were chosen. For each section, three images were recorded under the same conditions. The Image-Pro Plus 6.0 software was used to automatically read out the measurement values. The data of MBP-like immunoreactivity (IOD, integrated optical density) were expressed as percent of Control group.

Statistical analysis. SPSS17.0 (IBM Corp., Armonk, NY, USA) was used to analyze all the data which were expressed as mean \pm SD. The Shapiro-Wilk test was used for normality of data distribution. Independent student $\mathrm{t}$-test was used to compare mean values of two groups. The significant threshold was set at 0.05 .

Received: 10 April 2020; Accepted: 29 October 2020

Published online: 10 November 2020

\section{References}

1. Allen, N. J. \& Lyons, D. A. Glia as architects of central nervous system formation and function. Science 362, 181-185 (2018).

2. Allen, N. J. \& Barres, B. A. Neuroscience: glia-more than just brain glue. Nature 457, 675-677 (2009).

3. Oberheim, N. A. et al. Uniquely hominid features of adult human astrocytes. J. Neurosci. 29, 3276-3287 (2009).

4. Foster, A. Y., Bujalka, H. \& Emery, B. Axoglial interactions in myelin plasticity: evaluating the relationship between neuronal activity and oligodendrocyte dynamics. Glia 67, 2038-2049 (2019).

5. Murai, K. K. \& Pasquale, E. B. Eph receptors and ephrins in neuron-astrocyte communication at synapses. Glia 59, 1567-1578 (2011).

6. Schousboe, A., Scafidi, S., Bak, L. K., Waagepetersen, H. S. \& McKenna, M. C. Glutamate metabolism in the brain focusing on astrocytes. Adv. Neurobiol. 11, 13-30 (2014).

7. Miller, R. H. Regulation of oligodendrocyte development in the vertebrate CNS. Prog. Neurobiol. 67, 451-467 (2002).

8. Xiao, L. et al. Rapid production of new oligodendrocytes is required in the earliest stages of motor-skill learning. Nat. Neurosci. 19, 1210-1217 (2016).

9. Xu, H. et al. Evaluation of neuron-glia integrity by in vivo proton magnetic resonance spectroscopy: implications for psychiatric disorders. Neurosci. Biobehav. Rev. 71, 563-577 (2016).

10. Moffett, J. R., Ross, B., Arun, P., Madhavarao, C. N. \& Namboodiri, A. M. N-Acetylaspartate in the CNS: from neurodiagnostics to neurobiology. Prog. Neurobiol. 81, 89-131 (2007).

11. Maddock, R. J. \& Buonocore, M. H. MR spectroscopic studies of the brain in psychiatric disorders. Curr. Top. Behav. Neurosci. 11, 199-251 (2012).

12. Cai, K. et al. Magnetic resonance imaging of glutamate. Nat. Med. 18, 302-306 (2012).

13. George, E. D., Bordner, K. A., Elwafi, H. M. \& Simen, A. A. Maternal separation with early weaning: a novel mouse model of early life neglect. BMC Neurosci. 11, 123 (2010).

14. Strüber, N., Strüber, D. \& Roth, G. Impact of early adversity on glucocorticoid regulation and later mental disorders. Neurosci. Biobehav. Rev. 38, 17-37 (2014).

15. Haris, M. et al. Imaging of glutamate neurotransmitter alterations in Alzheimer's disease. NMR Biomed. 26, 386-391 (2013).

16. Crescenzi, R. et al. In vivo measurement of glutamate loss is associated with synapse loss in a mouse model of tauopathy. NeuroImage 101, 185-192 (2014).

17. Pépin, J. et al. In vivo imaging of brain glutamate defects in a knock.in mouse model of Huntington's disease. NeuroImage 139, 53-64 (2016).

18. Davis, K. A. et al. Glutamate imaging (GluCEST) lateralizes epileptic foci in nonlesional temporal lobe epilepsy. Sci. Transl. Med. 7, 309ra161 (2015).

19. O'Grady, K. P. et al. Glutamate sensitive imaging and evaluation of cognitive impairment in multiple sclerosis. Mult. Scler. 25, 1580-1592 (2019).

20. Dula, A., Pawate, S., Lyttle, B., Conrad, B. \& Smith, S. Measures of glutamate using chemical exchange saturation transfer (CEST) MRI related to cognition. Neurology 86(16 suppl), P4.135 (2016).

21. Roalf, D. et al. Glutamate imaging (GLUCEST) reveals lower brain glucest contrast in patients on the psychosis spectrum. Schizophr. Bull. 43(suppl 1), S49-S49 (2017).

22. Bagga, P. et al. Mapping the alterations in glutamate with GluCEST MRI in a mouse model of dopamine deficiency. J. Neurochem. 139, 432-439 (2016).

23. Hiser, J. \& Koenigs, M. The multifaceted role of the ventromedial prefrontal cortex in emotion, decision making, social cognition, and psychopathology. Biol. Psychiatry 83, 638-647 (2018).

24. Liu, W. et al. The role of neural plasticity in depression: from hippocampus to prefrontal cortex. Neural Plast. 2017, 6871089 (2017).

25. Penadés, R., Franck, N., González-Vallespí, L. \& Dekerle, M. Neuroimaging studies of cognitive function in schizophrenia. Adv. Exp. Med. Biol. 1118, 117-134 (2019).

26. Alexander, A. L., Lee, L. E., Lazar, M. \& Field, A. S. Diffusion tensor imaging of the brain. Neurotherapeutics 4, 316-329 (2007).

27. Bak, L. K., Schousboe, A. \& Waagepetersen, H. S. The glutamate/GABA-glutamine cycle: aspects of transport, neurotransmitter homeostasis and ammonia transfer. J. Neurochem. 98, 641-653 (2006).

28. Schousboe, A. \& Waagepetersen, H. S. Role of astrocytes in glutamate homeostasis: implications for excitotoxicity. Neurotox. Res. 8, 221-225 (2005).

29. Walls, A. B., Waagepetersen, H. S., Bak, L. K., Schousboe, A. \& Sonnewald, U. The glutamine-glutamate/GABA cycle: function, regional differences in glutamate and GABA production and effects of interference with GABA metabolism. Neurochem. Res. 40, 402-409 (2015).

30. Pellerin, L. \& Magistretti, P. J. Glutamate uptake stimulates $\mathrm{Na}+, \mathrm{K}+$-ATPase activity in astrocytes via activation of a distinct subunit highly sensitive to ouabain. J. Neurochem. 69, 2132-2137 (1997).

31. Schousboe, A. Role of astrocytes in the maintenance and modulation of glutamatergic and GABAergic neurotransmission. Neurochem. Res. 28, 347-352 (2003).

32. Francis, J. S. et al. N-acetylaspartate supports the energetic demands of developmental myelination via oligodendroglial aspartoacylase. Neurobiol. Dis. 96, 323-334 (2016). 
33. Bhakoo, K. K., Craig, T. J. \& Styles, P. Developmental and regional distribution of asparto-acylase in rat brain tissue. J. Neurochem. 79, 211-220 (2001).

34. Lu, Q. R. et al. Common developmental requirement for Olig function indicates a motor neuron/oligodendrocyte connection. Cell 109, 75-86 (2002).

35. Zhou, Q. \& Anderson, D. J. The bHLH transcription factors OLIG2 and OLIG1 couple neuronal and glial subtype specification. Cell 109, 61-73 (2002).

36. Emery, B. et al. Myelin gene regulatory factor is a critical transcriptional regulator required for CNS myelination. Cell 138, 172-185 (2009).

37. Murthy, S. et al. Perineuronal nets, inhibitory interneurons, and anxiety-related ventral hippocampal neuronal oscillations are altered by early life adversity. Biol. Psychiatry 85, 1011-1020 (2019).

38. Haris, M. et al. High resolution mapping of modafinil induced changes in glutamate level in rat brain. PLoS ONE 9, e103154 (2014).

39. Lowy, M. T., Gault, L. \& Yamamoto, B. K. Adrenalectomy attenuates stress-induced elevations in extracellular glutamate concentrations in the hippocampus. J. Neurochem. 61, 1957-1960 (1993).

40. Moghaddam, B. Stress preferentially increases extraneuronal levels of excitatory amino acids in the prefrontal cortex: comparison to hippocampus and basal ganglia. J. Neurochem. 60, 1650-1657 (1993).

41. Gilad, G. M., Gilad, V. H., Wyatt, R. J. \& Tizabi, Y. Region-selective stress-induced increase of glutamate uptake and release in rat forebrain. Brain Res. 525, 335-338 (1990).

42. Cholet, N., Pellerin, L., Magistretti, P. J. \& Hamel, E. Similar peri-synaptic glial localization for the Na+/K+-ATPase alpha 2 subunit and the glutamate transporters GLAST and GLT-1 in the rat somatosensory cortex. Cereb. Cortex 12, 515-525 (2002).

43. Roberts, R. C., Roche, J. K. \& McCullumsmith, R. E. Localization of excitatory amino acid transporters EAAT1 and EAAT2 in human postmortem cortex: a light and electron microscopic study. Neuroscience 277, 522-540 (2014).

44. Bauer, D. E. et al. The glutamate transporter, GLAST, participates in a macromolecular complex that supports glutamate metabolism. Neurochem. Int. 61, 566-574 (2012).

45. Genda, E. N. et al. Co-compartmentalization of the astroglial glutamate transporter, GLT-1, with glycolytic enzymes and mitochondria. J. Neurosci. 31, 18275-18288 (2011).

46. Matos, M., Augusto, E., Agostinho, P., Cunha, R. A. \& Chen, J. F. Antagonistic interaction between adenosine A2A receptors and $\mathrm{Na}+/ \mathrm{K}+$-ATPase- $\alpha 2$ controlling glutamate uptake in astrocytes. J. Neurosci. 33, 18492-18502 (2013).

47. Rose, E. M. et al. Glutamate transporter coupling to Na/K-ATPase. J. Neurosci. 29, 8143-8155 (2009).

48. Nowak, B. et al. Alterations in hippocampal calcium-binding neurons induced by stress models of depression: a preliminary assessment. Pharmacol. Rep. 62, 1204-1210 (2010).

49. Zadrozna, M. et al. Different pattern of changes in calcium binding proteins immunoreactivity in the medial prefrontal cortex of rats exposed to stress models of depression. Pharmacol. Rep. 63, 1539-1546 (2011).

50. Kobayashi, M., Hayashi, Y., Fujimoto, Y. \& Matsuoka, I. Decreased parvalbumin and somatostatin neurons in medial prefrontal cortex in BRINP1-KO mice. Neurosci. Lett. 683, 82-88 (2018).

51. Berkowicz, S. R. et al. Brinp1 (-/-) mice exhibit autism-like behavior, altered memory, hyperactivity and increased parvalbuminpositive cortical interneuron density. Mol. Autism 7, 22 (2016).

52. Maier, H., Wang-Eckhardt, L., Hartmann, D., Gieselmann, V. \& Eckhardt, M. N-Acetylaspartate synthase deficiency corrects the myelin phenotype in a Canavan disease mouse model but does not affect survival time. J. Neurosci. 35, 14501-14516 (2015).

53. Zatorre, R. J., Fields, R. D. \& Johansen-Berg, H. Plasticity in gray and white: neuroimaging changes in brain structure during learning. Nat. Neurosci. 15, 528-536 (2012).

54. Carlyle, B. C. et al. Maternal separation with early weaning: a rodent model providing novel insights into neglect associated developmental deficits. Dev. Psychopathol. 24, 1401-1416 (2012).

55. Makinodan, M., Rosen, K. M., Ito, S. \& Corfas, G. A critical period for social experience-dependent oligodendrocyte maturation and myelination. Science 337, 1357-1360 (2012).

56. Zhang, H. et al. The recovery trajectory of adolescent social defeat stress-induced behavioral, (1)H-MRS metabolites and myelin changes in Balb/c mice. Sci. Rep. 6, 27906 (2016).

57. Luo, M. et al. Differential susceptibility and vulnerability of brain cells in C57BL/6 mouse to mitochondrial dysfunction induced by short-term cuprizone exposure. Front. Neuroanat. 14, 30 (2020).

58. Hoshino, H. \& Kubota, M. Canavan disease: Clinical features and recent advances in research. Pediatr. Int. 56, 477-483 (2014).

59. Wise, S. P. Forward frontal fields: phylogeny and fundamental function. Trend Neurosci. 31, 599-608 (2008).

60. Shao, Y. et al. Chronic social isolation decreases glutamate and glutamine levels and induces oxidative stress in the rat hippocampus. Behav. Brain Res. 282, 201-208 (2015).

61. Zhuang, Z. et al. Mapping the changes of glutamate using glutamate chemical exchange saturation transfer (GluCEST) technique in a traumatic brain injury model: a longitudinal pilot study. ACS Chem. Neurosci. 10, 649-657 (2019).

62. Cakmakci, H., Pekcevik, Y., Yis, U., Unalp, A. \& Kurul, S. Diagnostic value of proton MR spectroscopy and diffusion-weighted MR imaging in childhood inherited neurometabolic brain diseases and review of the literature. Eur. J. Radiol. 74, e161-e171 (2010).

63. Zhang, L., Xu, S., Huang, Q. \& Xu, H. N-acetylcysteine attenuates the cuprizone-induced behavioral changes and oligodendrocyte loss in male C57BL/7 mice via its anti-inflammation actions. J. Neurosci. Res. 96, 803-816 (2018).

\section{Author contributions}

H.Z., W.W., Q.H., and H.X. conceived the idea. H.Z., Z.S., Z.D., and X.C. performed DTI, GluCEST, and 1H-MRS scanning and analyzed the neuroimaging data. G.Y., X.Z., R.W., and H.X. instructed the neuroimaging experiments and interpreted the neuroimaging data. H.Z., Z.Y., and S.X. performed behavioral tests, immunohistochemical staining, RT-PCR, and Western-blot experiments. H.Z and H.X. interpreted and integrated all the data. Q.H., R.W., and H.X. supervised the research. H.Z. and H.X. wrote the manuscript with input from all coauthors. All authors approved the final version of the manuscript.

\section{Competing interests}

The authors declare no competing interests.

\section{Additional information}

Supplementary information is available for this paper at https://doi.org/10.1038/s41598-020-76640-y.

Correspondence and requests for materials should be addressed to H.X.

Reprints and permissions information is available at www.nature.com/reprints.

Publisher's note Springer Nature remains neutral with regard to jurisdictional claims in published maps and institutional affiliations. 
(c) (i) Open Access This article is licensed under a Creative Commons Attribution 4.0 International cc) License, which permits use, sharing, adaptation, distribution and reproduction in any medium or format, as long as you give appropriate credit to the original author(s) and the source, provide a link to the Creative Commons licence, and indicate if changes were made. The images or other third party material in this article are included in the article's Creative Commons licence, unless indicated otherwise in a credit line to the material. If material is not included in the article's Creative Commons licence and your intended use is not permitted by statutory regulation or exceeds the permitted use, you will need to obtain permission directly from the copyright holder. To view a copy of this licence, visit http://creativecommons.org/licenses/by/4.0/.

(C) The Author(s) 2020 